\title{
Short distance neutrino oscillations with Borexino
}

A. Caminata ${ }^{1, a}$, M. Agostini' ${ }^{2}$, K. Altenmüller ${ }^{2}$, S. Appel ${ }^{2}$, G. Bellini' ${ }^{3}$, J. Benziger ${ }^{4}$, N. Berton ${ }^{5}$, D. Bick ${ }^{6}$, G. Bonfini ${ }^{7}$, D. Bravo ${ }^{8}$, B. Caccianiga ${ }^{3}$, F. Calaprice ${ }^{9,10}$, P. Cavalcante ${ }^{7}$, A. Chepurnov ${ }^{11}$, M. Cribier ${ }^{5}$, D. D'Angelo ${ }^{3}$, S. Davini ${ }^{10}$, A. Derbin ${ }^{12}$, L. di Noto ${ }^{1}$, M. Durero ${ }^{5}$, A. Empl ${ }^{13}$, A. Etenko ${ }^{14,15}$, S. Farinon ${ }^{1}$, V. Fischer ${ }^{5}$, K. Fomenko ${ }^{16}$, D. Franco ${ }^{17}$, F. Gabriele ${ }^{7}$, J. Gaffiot ${ }^{5}$, C. Galbiati ${ }^{9}$, C. Ghiano ${ }^{7}$, M. Giammarchi ${ }^{3}$, M. Göger-Neff ${ }^{2}$, A. Goretti ${ }^{9}$, M. Gromov ${ }^{11}$, C. Hagner ${ }^{6}$, Th. Houdy ${ }^{5}$, E. Hungerford ${ }^{19}$, Aldo Ianni $^{7}$, Andrea Ianni ${ }^{9}$, N. Jonquères ${ }^{20}$, M. Kaiser ${ }^{6}$, V. Kobychev ${ }^{21}$, D. Korablev ${ }^{16}$, G. Korga ${ }^{19}$, D. Kryn ${ }^{17}$, T. Lachenmaier ${ }^{22}$, T. Lasserre ${ }^{5}$, M. Laubenstein ${ }^{7}$, B. Lehnert ${ }^{23}$, J. Link ${ }^{8}$, E. Litvinovich ${ }^{14,15}$, F. Lombardi ${ }^{7}$, P. Lombardi ${ }^{3}$, L. Ludhova ${ }^{3}$, G. Lukyanchenko ${ }^{14}$, I. Machulin ${ }^{14,15}$, W. Maneschg ${ }^{24}$, S. Marcocci ${ }^{1,10}$, J. Maricic ${ }^{25}$, G. Mention ${ }^{5}$, E. Meroni ${ }^{3}$,

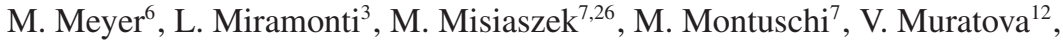
R. Musenich ${ }^{1}$, B. Neumair ${ }^{2}$, L. Oberauer ${ }^{2}$, M. Obolensky ${ }^{17}$, F. Ortica ${ }^{27}$, M. Pallavicini ${ }^{1}$, L. Papp ${ }^{2}$, L. Perasso ${ }^{1}$, A. Pocar $^{28}$, G. Ranucci ${ }^{3}$, A. Razeto ${ }^{7}$, A. Re ${ }^{3}$, A. Romani ${ }^{27}$, N. Rossi ${ }^{7}$, S. Schönert ${ }^{2}$, L. Scola ${ }^{5}$, H. Simgen ${ }^{24}$, M. Skorokhvatov ${ }^{14,15}$, O. Smirnov ${ }^{16}$, A. Sotnikov ${ }^{16}$, S. Sukhotin ${ }^{14}$, Y. Suvorov ${ }^{7,14,29}$, R. Tartaglia ${ }^{7}$, G. Testera ${ }^{1}$, C. Veyssière $^{5}$, M. Vivier $^{5}$, R.B. Vogelaar ${ }^{8}$, F. von Feilitzsch ${ }^{2}$, H. Wang ${ }^{29}$, J. Winter ${ }^{30}$, M. Wojcik ${ }^{26}$, M. Wurm ${ }^{30}$, O. Zaimidoroga ${ }^{16}$, S. Zavatarelli ${ }^{1}$, K. Zuber ${ }^{23}$, and G. Zuzel ${ }^{26}$

${ }^{1}$ Dipartimento di Fisica, Università degli Studi e INFN, 16146 Genova, Italy

${ }^{2}$ Physik-Department and Excellence Cluster Universe, Technische Universität München, 85748 Garching, Germany

${ }^{3}$ Dipartimento di Fisica, Università degli Studi e INFN, 20133 Milano, Italy

${ }^{4}$ Chemical Engineering Department, Princeton University, Princeton, New Jersey 08544, USA

${ }^{5}$ Commissariat à l'Energie Atomique et aux Energies Alternatives, Centre de Saclay, IRFU, 91191 Gif-sur-Yvette, France

${ }^{6}$ Institut für Experimentalphysik, Universität Hamburg, 22761 Hamburg, Germany

${ }^{7}$ INFN Laboratori Nazionali del Gran Sasso, 67100 Assergi, Italy

${ }^{8}$ Physics Department, Virginia Polytechnic Institute and State University, Blacksburg, Virginia 24061, USA

${ }^{9}$ Physics Department, Princeton University, Princeton, New Jersey 08544, USA

${ }^{10}$ Gran Sasso Science Institute (INFN), 67100 L'Aquila, Italy

${ }^{11}$ Lomonosov Moscow State University Skobeltsyn Institute of Nuclear Physics, 119234 Moscow, Russia

${ }^{12}$ St Petersburg Nuclear Physics Institute, 188350 Gatchina, Russia

${ }^{13}$ Department of Physics, University of Houston, Houston, Texas 77204, USA

${ }^{14}$ NRC Kurchatov Institute, 123182 Moscow, Russia

${ }^{15}$ National Research Nuclear University MEPhI (Moscow Engineering Physics Institute), 115409 Moscow, Russia

${ }^{16}$ Joint Institute for Nuclear Research, 141980 Dubna, Russia

${ }^{17}$ APC, Université Paris Diderot, CNRS/IN2P3,CEA/Irfu, Observatoire de Paris, Sorbonne Paris Cité, 75205 Paris Cedex 13, France

ae-mail: alessio.caminata@ge.infn.it

(C) The Authors, published by EDP Sciences. This is an Open Access article distributed under the terms of the Creative Commons Attribution License 4.0 (http://creativecommons.org/licenses/by/4.0/). 
${ }^{18}$ Institut für Experimentalphysik, Universität Hamburg, 22761 Hamburg, Germany

${ }^{19}$ Department of Physics, University of Houston, Houston, Texas 77204, USA

${ }^{20}$ Commissariat à l'Energie Atomique et aux Energies Alternatives, Centre de Saclay, DEN/DM2S/SEMT/BCCR, 91191 Gif-sur-Yvette, France

${ }^{21}$ Kiev Institute for Nuclear Research, 03680 Kiev, Ukraine

${ }^{22}$ Kepler Center for Astro and Particle Physics, Universitt Tübingen, 72076 Tübingen, Germany

${ }^{23}$ Department of Physics, Technische Universität Dresden, 01062 Dresden, Germany

${ }^{24}$ Max-Planck-Institut für Kernphysik, 69117 Heidelberg, Germany

${ }^{25}$ Department of Physics and Astronomy, University of Hawai'i, Honolulu, HI 96822, USA

${ }^{26}$ M. Smoluchowski Institute of Physics, Jagiellonian University, 30059 Krakow, Poland

${ }^{27}$ Dipartimento di Chimica, Biologia e Biotecnologie, Universita“ degli Studi e INFN, 06123 Perugia, Italy

${ }^{28}$ Physics Department, University of Massachusetts, Amherst, Massachusetts 01003, USA

${ }^{29}$ Physics and Astronomy Department, University of California Los Angeles (UCLA), Los Angeles, California 90095, USA

${ }^{30}$ Institut für Physik, Johannes Gutenberg Universität Mainz, 55122 Mainz, Germany

\begin{abstract}
The Borexino detector has convincingly shown its outstanding performances in the low energy, sub-MeV regime through its unprecedented accomplishments in the solar and geo-neutrinos detection. These performances make it the ideal tool to accomplish a state-of-the-art experiment able to test unambiguously the long-standing issue of the existence of a sterile neutrino, as suggested by the several anomalous results accumulated over the past two decades, i.e. the outputs of the LSND and Miniboone experiments, the results of the source calibration of the two Gallium solar neutrino experiments, and the recently hinted reactor anomaly. The SOX project will exploit two sources, based on Chromium and Cerium, respectively, which deployed under the experiment, in a location foreseen on purpose at the time of the construction of the detector, will emit two intense beams of neutrinos $(\mathrm{Cr})$ and anti-neutrinos $(\mathrm{Ce})$. Interacting in the active volume of the liquid scintillator, each beam would create an unmistakable spatial wave pattern in case of oscillation of the $v_{e}\left(\right.$ or $\left.\bar{v}_{e}\right)$ into the sterile state: such a pattern would be the smoking gun proving the existence of the new sterile member of the neutrino family. Otherwise, its absence will allow setting a very stringent limit on its existence.
\end{abstract}

\title{
1. Introduction
}

Neutrino flux measurements by real-time detectors have confirmed the neutrino oscillations between its three known flavor states. Borexino, a world-class example of this kind of detector, has measured different components of the solar neutrinos [1,2], including the $p p$ neutrinos, which are produced in the reaction that releases $99.77 \%$ of the Sun energy [3].

In the near future, Borexino aims to investigate the existence of neutrino oscillations in sterile states (beyond the known $v_{e}, v_{\mu}$ and $v_{\tau}$ states) which are detectable as a deficit in a neutrino flux. The Short distance neutrino Oscillations with BoreXino project will explore the $\Delta m_{14}^{2}$ region of $\sim 1 \mathrm{eV}^{2}$ [4].

\section{The Borexino detector}

Borexino is currently installed in Hall C of the Laboratori Nazionali del Gran Sasso (LNGS) in Italy. Its design is based on the principle of graded shielding with the inner scintillating core surrounded by concentric vessels of decreasing radio purity from inside to outside (Fig. 1). The active scintillator is a solution of PPO (2,5-diphenyloxazole), which 


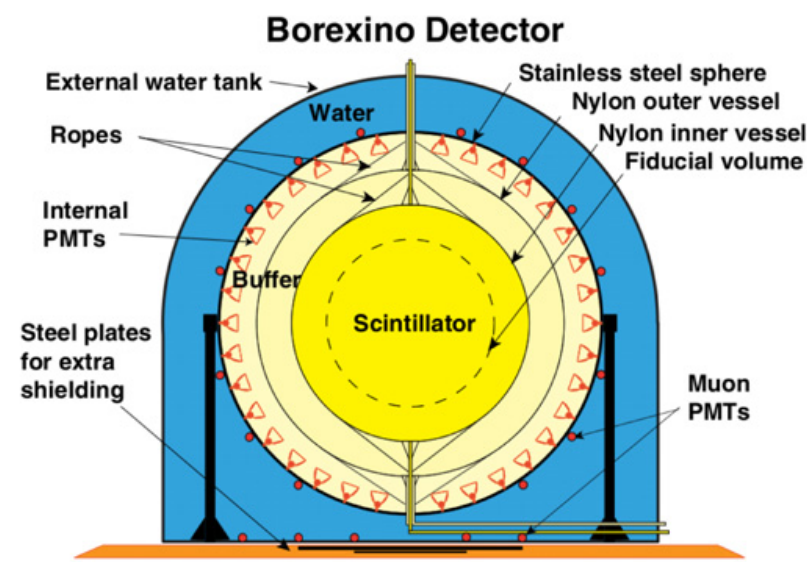

Figure 1. A schematic view of the Borexino detector.

is a wavelength-shifter, at a concentration of $1.5 \mathrm{~g} / 1$ in pseudocumene (PC, 1,2,4trimethylbenzene). The scintillator is contained in a $125 \mu \mathrm{m}$ thick spherical inner nylon vessel (IV) with $4.25 \mathrm{~m}$ radius. The IV is surrounded by a passive shield made by PC in which a small quantity of DMP (dimethylphthalate) is added to inhibit the scintillation process. The scintillation light is collected by 22128 inch PMTs. The scintillator and the PMTs are located inside a 18-meter-diameter stainless steel sphere which is contained in a water Čerenkov tank acting as active muon ${ }^{1}$ veto (efficiency greater than $99.99 \%$ ).

The light yield is about 500 photoelectrons/MeV with a threshold of about $60 \mathrm{keV}$. Since scintillation light coming from nuclear decays inside the inner vessel is indistinguishable from the neutrino elastic-scattering off electrons in PC, an extreme radiopurity level must be maintained. Thanks to specific scintillator purification activities, an unprecedented level of several $10^{-19}$ grams of ${ }^{238} U$ and ${ }^{232} \mathrm{Th}$ per gram of scintillator is reached. From the beginning of data taking, four major calibration campaigns with different kinds of radiation sources $(\alpha$, $\beta, \gamma$ and neutrons) have been taken place. The spatial resolution of the detector is about $10 \mathrm{~cm}$ at the energy of $1 \mathrm{MeV}$ while the energy resolution is about 5\% [1].

\section{The SOX project}

The SOX experiment [4] aims at the complete confirmation or at a clear disproof of the so-called neutrino anomalies, a set of circumstantial evidences of electron neutrino disappearance observed at LSND [5], MiniBoone [6], with nuclear reactors [7] and with solar neutrino Gallium detectors [8]. If successful, SOX will demonstrate the existence of sterile neutrino components and will open a brand new era in fundamental particle physics and cosmology. A solid signal would mean the discovery of the first particles beyond the Standard Electroweak Model and would have profound implications in our understanding of the Universe and of fundamental particle physics. In case of a negative result, it is able to close a long standing debate about the reality of the neutrino anomalies, would probe the existence of new physics in low energy neutrino interactions, would provide a measurement of neutrino

\footnotetext{
1 The Gran Sasso d'Italia mountains, under which the LNGS are located, provide a 3600 meters of equivalent water shielding against cosmic rays.
} 
magnetic moment, and would yield a superb energy calibration for Borexino which will be very beneficial for future high-precision solar neutrino measurements.

The Borexino detector is placed over a small tunnel designed to deploy in it neutrino sources to calibrate the experiment. The first stage of the experiment (SOX-A) will consist in the deployment of high-intensity $v_{e}\left({ }^{51} \mathrm{Cr}\right)$ and $\bar{v}_{e}\left({ }^{144} \mathrm{Ce}\right)$ sources in the pit, at a distance of $8.25 \mathrm{~m}$ from the detector's center.

\section{$3.1{ }^{51} \mathrm{Cr}$ neutrino source}

A first option to test the existence of sterile neutrinos will be placing a $10 \mathrm{MCi}{ }^{51} \mathrm{Cr}$ source in the pit. The ${ }^{51} \mathrm{Cr}$ source will be produced by irradiating a large sample of $38 \%-{ }^{50} \mathrm{Cr}$-enriched material in a nuclear reactor. Since a high flux nuclear reactor is needed, the enrichment could be done in High-Flux Isotope Reactor (HFIR) in Oak Ridge National Laboratory USA or in the Mayak reactor in Russia. ${ }^{51} \mathrm{Cr}$ decays via electron capture into ${ }^{51} \mathrm{~V}$ emitting two neutrino lines of $750 \mathrm{keV}(90 \%)$ and $430 \mathrm{keV}(10 \%)$. Since the energy is very similar to the energy of ${ }^{7} \mathrm{Be}$ neutrinos (or close to the pp cut-off for the less dominant component), Borexino has already proven its capability to detect neutrinos of these energies. Due to ${ }^{51} \mathrm{Cr}$ short half-life $\left(t_{1 / 2}=27.7\right.$ days $)$, a very quick transportation to LNGS is needed.

\section{$3.2{ }^{144} \mathrm{Ce}$ anti-neutrino source}

A recently developed plan is to deploy a $\approx 150 \mathrm{kCi}{ }^{144} \mathrm{Ce}$ antineutrino source in the pit. Antineutrinos are detected by means on inverse beta decay (IBD, threshold $1.8 \mathrm{MeV}$ ) on protons. The low radioactivity and the clean tag offered by the space-time coincidence between the prompt $e^{+}$and the subsequent neutron capture ( $\tau=254 \mu s$ [9]) make accidental background essentially zero. The ${ }^{144} \mathrm{Ce}$ source $\beta$-decays to ${ }^{144} \operatorname{Pr}\left(t_{1 / 2}=296\right.$ days $)$ which rapidly $(\tau=$ $17 \mathrm{~min}) \beta$-decays to ${ }^{144} \mathrm{Nd}$ emitting $\bar{v}_{e} \mathrm{~s}$. The detection threshold for IBD is $1.8 \mathrm{MeV}$, matching adequately the energy of the $\bar{\nu}_{e}$ emitted by the source (the end point of the ${ }^{144} \mathrm{Pr}$ is about $3 \mathrm{MeV}$ ).

\subsection{Calorimetry and data analysis}

The sources, with the proper shielding, will be placed in water-flow calorimeters to measure their activities. For the ${ }^{51} \mathrm{Cr}$ source, due to the shorter lifetime, calorimetry will be performed continuously during the data-taking while the source is deployed under the detector. For the ${ }^{144} \mathrm{Ce}$ source, the measurement will be performed before inserting the source under Borexino and at the end of data-taking, when the source will be removed from the pit. Calorimeters design is mostly finalized and a prototype with resistive heaters will soon be starting acceptance tests in the first part of 2015.

Borexino can study short distance neutrino oscillations in two ways. The first way is the standard disappearance technique (rate technique): if oscillations occur, the total count rate is lower than that expected without oscillations. The second way is based on an oscillometry measurement within the detector volume (rate+shape technique). If the values of $\Delta m_{41}^{2}$ are of the order of $1 \mathrm{eV}^{2}$ and the neutrino energy is of the order of $1 \mathrm{MeV}$, the typical oscillation length is about few meters and, being the spatial resolution about $10 \mathrm{~cm}$ (Sect. 2), the resulting oscillations waves can be directly seen in Borexino [4]. This technique will allow a direct measurement of $\Delta m_{41}^{2}$ and $\theta_{41}$, investigating most of the anomaly phase space (Fig. 2). The best fit values for the current allowed phase space will be investigated to generate a discovery/exclusion signal at $5 \sigma$ [10]. In parallel with the sterile neutrino search, some precision measurements will be done. For example a much lower energy $(\approx 1 \mathrm{MeV})$ 


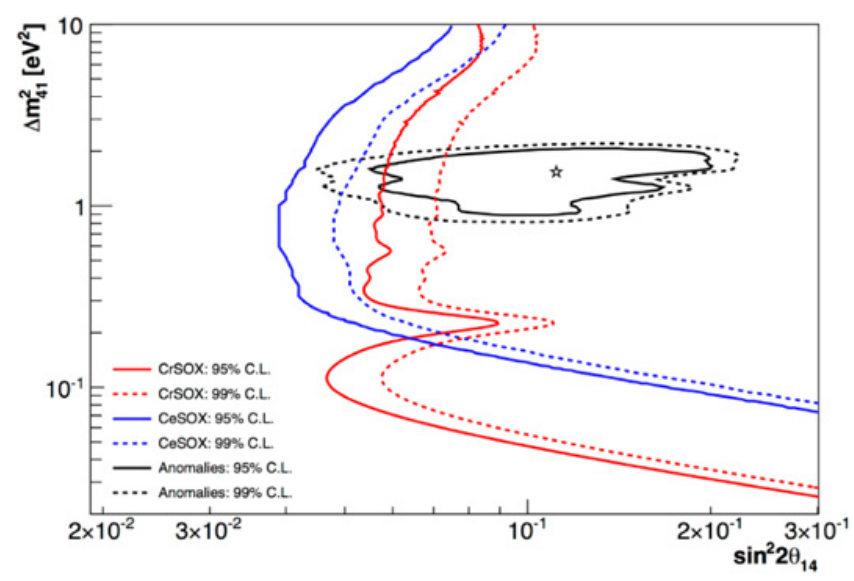

Figure 2. Allowed parameter space accordingly to one of the latest review of global fits [10] and region of space covered by SOX-A experiment.

measurement of the Weinberg angle, $g_{A}$ and $g_{V}$ parameters at low energies and studies of the neutrino magnetic moment.

The plan for the mid-term future is to insert a $75 \mathrm{kCi}{ }^{144} \mathrm{Ce}$ source inside the water tank (SOX-B, 2017) and in the center of Borexino (SOX-C, later). This projects require a refurbishment of the detector and a more challenging active cooling of the source and will be performed after the completion of the Borexino solar physics program. With SOX-B and SOX-C it will be possible to investigate the gallium+reactor anomaly $99 \%$-allowed phase space to $95 \%$ and $99 \%$ confidence level respectively.

\section{Conclusions}

The Borexino detector is an ideal detector to search for sterile neutrinos. Thanks to the high radio-purity and the low energy threshold, it is possible to test the existence of sterile neutrinos using both a neutrino and an anti-neutrino source. The data taking will start at the end of 2015 deploying a ${ }^{144} \mathrm{Ce}$ anti-neutrino generator in the pit under the Borexino detector and the first results will be expected in 2016.

\section{References}

[1] G. Bellini et al., Physical Review D 89, 112007 (2014)

[2] G. Bellini et al., Physical Review D 82, 033006 (2010)

[3] G. Bellini et al., Nature 512, 383 (2014)

[4] G. Bellini et al., Journal of High Energy Physics 38, 1 (2013)

[5] A. Aguilar et al., Physical Review D 64, 112007 (2001)

[6] A. Aguilar et al., Physical Review Letters 110, 161801 (2013)

[7] G. Mention et al., Physical Review D 83, 073006 (2011)

[8] C. Giunti et al., Physical Review C 83, 065504 (2011)

[9] G. Bellini et al., Physics Letters B 722, 295-300 (2013)

[10] C. Giunti et al., Physical Review D 88, 073008 (2013) 